\title{
Economical Deployable Optical Network Communication System for Space and Water
}

\author{
Shiv Vyas \\ Computer Engineering Department, \\ Sardar Vallabhbhai Patel Institute of Technology, Vasad \\ Gujarat Technological University, Ahmedabad
}

\begin{abstract}
The experiment by the Advanced Research Projects Agency in 1960 s led to the foundation of Internet. Internet has now become an integral part of our daily life. From then technologies have progressed from $2 \mathrm{G}, 3 \mathrm{G}, 4 \mathrm{G}, 5 \mathrm{G}$ and now even Visible Light. This paper focuses on LI-FI and Developing a new economical optical network communication system for space and water. Light Fidelity is known as LI-FI. LI-FI was first introduced to the world by German Psychist Harald Hass a professor at Edinburg University. [1]LI-Fi is Wireless Optical networking technology that uses Light Emitting Diode (LED) for Data-Communication. Li-Fi is claimed to be 100 times faster than WI-FI. LI-FI provides better bandwidth, efficiency and security than Wi-Fi. In Order for sound to travel there has to be something with molecules for it to travel through, sound needs molecules to vibrate for it to travel. On earth sound travels by vibrating air molecules. In space there are large empty space between planets and stars so there is nothing for molecules to vibrate as a result there is no sound in space. Sound can travel in water but at a speed of 4.3 times faster than in air and also the words and directions are deformed. Li-Fi uses Visible light spectrum and can be developed by blinking LED's at very high speed. Visible Light can travel in both space and water. Visible light can be used to develop an economical and convenient communication system for space and water.
\end{abstract}

\section{Keywords}

Li-Fi (Light Fidelity), Wi-Fi (Wireless Fidelity), Vacuum, Sound, Underwater, LED, Photo-Diode, Astronauts, Divers,Communications

\section{INTRODUCTION}

In Today's technological world communication is most important aspect of everyone's life. Communication is used for transfer of data, where data can be anything audio, video, etc. Communication can be of any type Human to human, machine to machine, human to machine. The term LI-FI was first introduced by Harald Hass a German professor of Mobile Communication at University of Edinburg, Scotland in year 2011 at a global TED Talk. He first Demonstrated LI-FI by flickering a single LED to transfer data. To prove his point, he also blocked the light and proved that light was the only source of data. Harald Hass is also the co-founder of PureLIFI, a company that develops Li-Fi, a visible light communications (VLC) technology for the use of visible light spectrum instead of radio frequencies (RFs) to enable wireless data communication.LI-FI can be used in areas where we require speed, security and efficiency. LI-FI has many applications such as in self-driving cars, medical science, airbus and many more.

\section{EXISTING SYSTEM} \subsection{Existing Communication System For} Space:

There is no sound in space as sound needs a medium to travel. In space the biggest problem with astronauts is of communication with each other. Visible light and some forms of electromagnetic radiations can travel in space. The most common form used for communication is Radio. For communication, helmets of astronauts are designed with a special inbuilt device. This device captures the voice of astronauts and converts it into to radio waves and this radio waves are sent to the fellow astronauts. On receiving the radio waves by the fellow astronaut, it is again converted back into sound which can be heard by a human. Radio waves are often thought of as a form of sound because of their use in this way, but radio waves are not sound waves, they are form of electromagnetic radiation analogous to visible light, and therefore can propagate through vacuum.

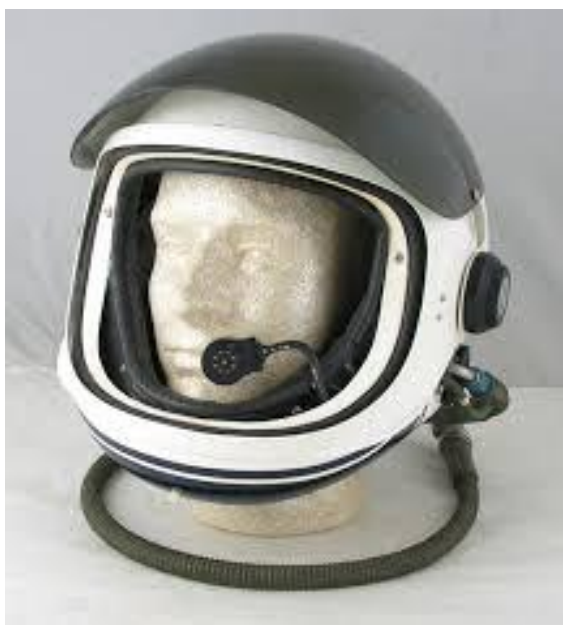

Fig: [1] Astronauts Helmet with Microphone and speaker

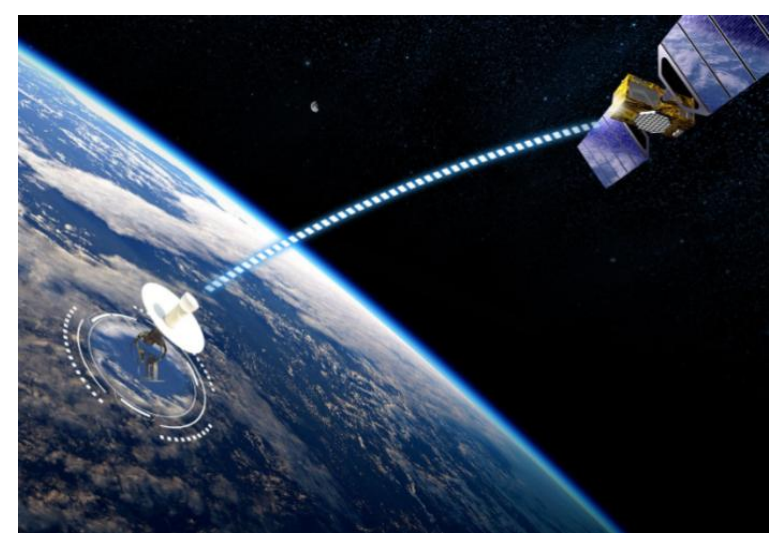

Fig: [2] Transfer of Data on earth from Spacecraft 
There is black coloured microphone attached near the mouth of astronaut which captures the voice of astronauts and is further converted into radio waves. The radio waves are received by others and heard with the speaker attached in the inner part of the helmet near the ears of astronauts.

Radio waves are also used for communication with spacecraft from earth. Each spacecraft has a transmitter and receiver for radio waves as well as a way of interpreting the information received and acting on it. Certain constraints are to be focused here such as the strength of radio waves get weakened when they reach earth, radio waves must be precisely reach on target. NASA has giant radio receivers which are used to collect information from space.

\subsection{Existing Communication System For Water.}

Sound can travel through water but it cannot be used by divers for communication as speed of sound increases by 4.3 times as that in air. Moreover, a human being cannot produce a sound that can travel in water as vocal cords of humans are not designed for such purpose. Radio signals will also not work effectively because radios cannot propagate to a desired distance in water.

Scuba Divers uses very basic and old method of communication with each other's. Divers are specially trained to use sign signals to communicate with each other. In general hand movement is made to produce a signal which is easily understood by other divers. Each Signal has a particular meaning such as to follow, air supply, no negative, etc.
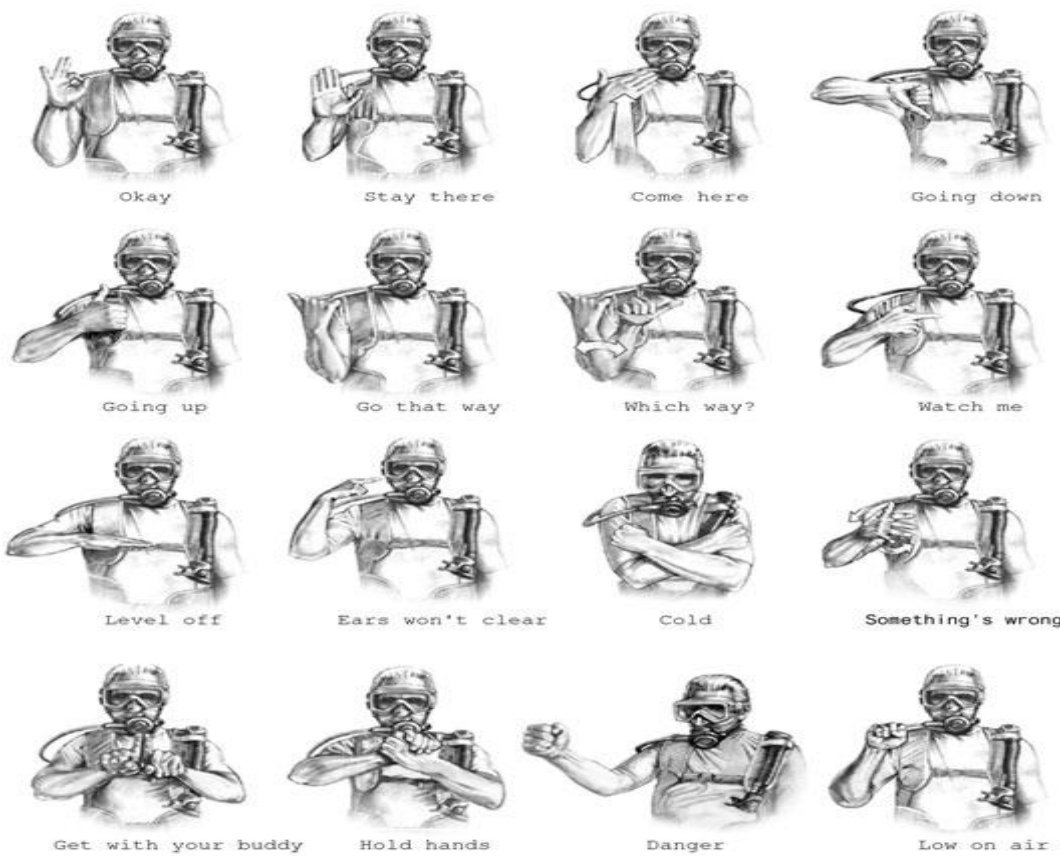

Fig: [3] Hand signals used by divers for communication

There is one more method involved for communication which is based on reading and writing. Special purpose writing pads are designed which allows divers to write under water. When one diver wants to communicate, he

writes it on the writing pad which is read by others and they can reply back in similar manner.

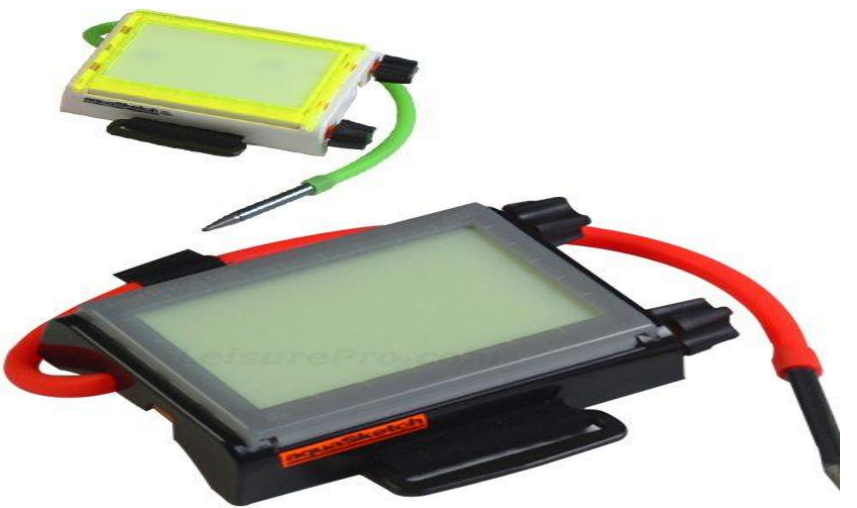

Fig.: [4] Underwater Writing Pad
As shown in figure no.[4] there is special screen attached on the pad which is visible in water and also a stylus is attached with the Writing pad which is used for writing. There are certain limitations for both these techniques such as both requires light, both requires a clear water to be used and cannot be used in murky waters. To overcome such limitations ultrasonic sound and SONAR(Sound Navigation and Ranging) are also used sometimes for communication with surface ship or even for submarine to submarine communication.

\section{PROPOSED SYSTEM}

The proposed system is based on visible light communication (VLC). [2] Visible light is the form in which electromagnetic radiations with wavelength in particular range are interpreted by human brain. The definition of Li-Fi can be stated as electromagnetic waves which are capable of being perceived by sight. Spectrum of visible light ranges from $380 \mathrm{~nm}$ to $750 \mathrm{~nm}$ which is found to be violet-bluish tone at $380 \mathrm{~nm}$ and distinctly red at $750 \mathrm{~nm}$. Under normal condition human being are not able to detect infrared light and ultraviolet light. Ultraviolet light which is beyond violet is perceived by certain animals such as birds, bees and fish while animals like snakes 
can see infrared light. Any system which is designed on the principle of $\mathrm{Li}-\mathrm{Fi}$ will have two major components:

1) A transmitter - LED

2)A Receiver - Photo Sensor

System 1
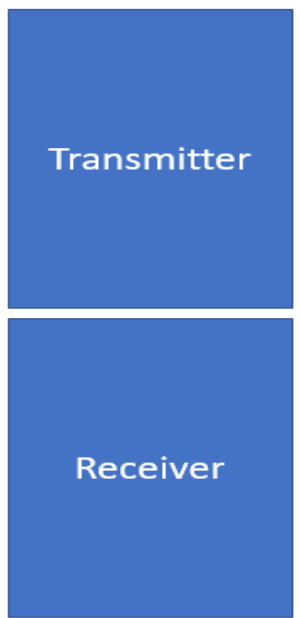

The logic behind it is that one works as a sender which is LED, it will transmit the data and other works as a receiver which is a photo diode having sensitivity towards light to receive the data.

Fig:[5] Block diagram of communication between two systems

The system is divided into two parts one sender section and one receiver section. When sender unit of one system will transmit the data, the receiver unit of the second system will be automatically invoked. Similarly, after processing the data the sender section of the second unit will respond back and the receiver section of the first system will be invoked.

\subsection{Hardware Configuration And Working}

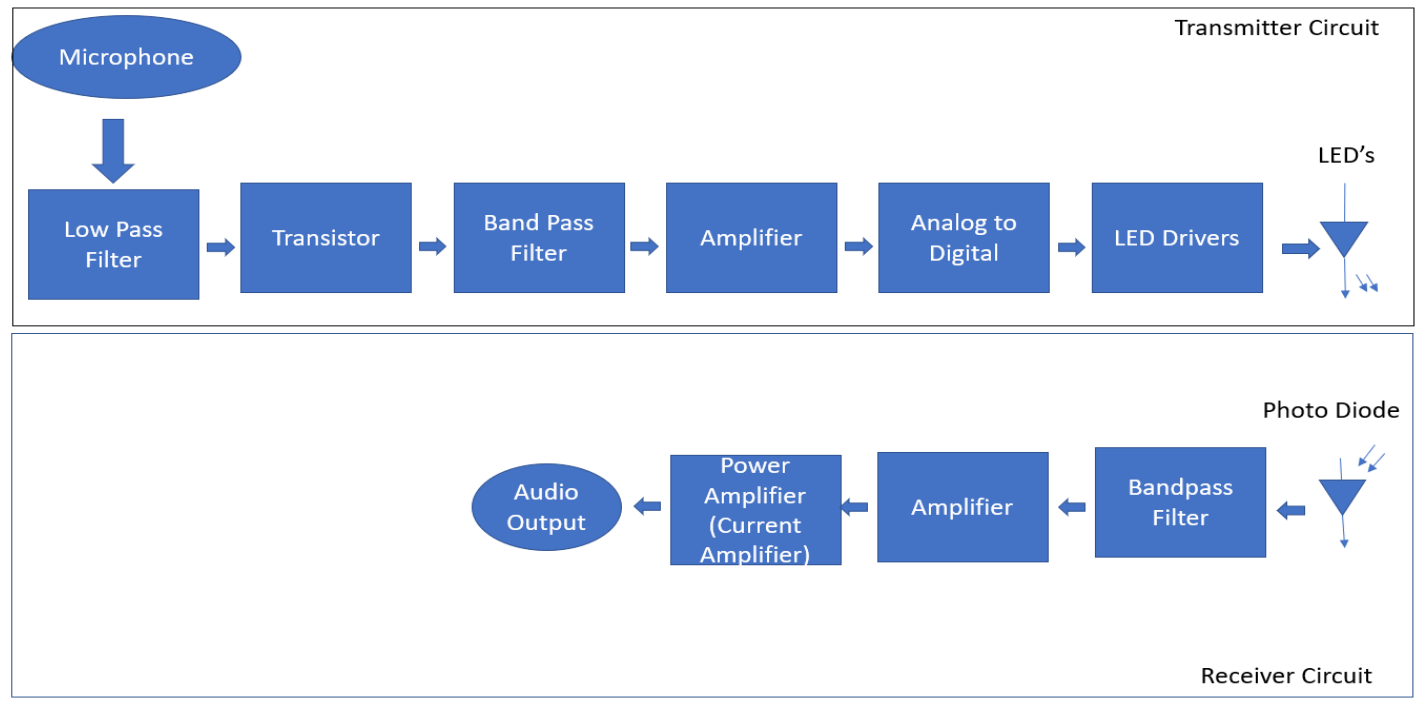

Fig:[6] Block Diagram of the optical network communication system using $\mathrm{Li}-\mathrm{Fi}$

\subsubsection{MICROPHONE}

It works as an input transducer. Its work is to convert the voice of human being into an electrical signal. There are two types of microphone one is passive microphone which will give output of only acoustics it absorbs and other is an active microphone which will also control an external power source.

\subsubsection{LOW-PASS FILTER}

Low pass filter is used to pass low frequency components of audio. Its job is to remove unwanted noise from the actual audio. The reason of using low pass filter is that if there any high frequency noise than it will not allow it.

\subsubsection{TRANSISTOR}

It is a three terminal device which can be used as an amplifier or a switch. It forms the basis of modern day electronic industry. BC 547 transistor can be used to convert sound in micro volts to few milli volts. Its job is to perform basic amplification.

\subsubsection{AMPLIFIER}

It is used to increase power of signals. TTL and CMOSS logic are matcher here. It will convert micro volts to volts. Its works to perform higher amplification. 


\subsubsection{ANALOG TO DIGITAL}

In electronics an Analog to Digital converter system is a system that converts an analog signal, such as sound picked by a microphone or light entering a digital camera, into a digital signal.

\subsubsection{LED Driver}

The main purpose of LED drivers is to regulate the power supplied to LED. LED driver is an electronic circuit which will provide power to the LED according to the input given to it.

\subsubsection{LED (Light Emitting Diode)}

One of the primary components of electronic industry is LED. It is a P-N junction diode, which emits light when in active state.

\subsubsection{Photo Diode}

The conversion of light into electrical signal takes place with help of photodiode. The electrical current is generated when photons are absorbed by photodiode.

\subsubsection{BONE-CONDUCTION SPEAKER}

It works as an output transducer. It used to hear the sound generated by the amplified signal.

\subsection{Configuration}

\subsubsection{Transmitter Section}

Figure No.:[7] shows Transmitter section circuit diagram for sending the data. Microphone will capture the voice and convert it into electrical signal. This electrical signal is provided as an input to the transistor BC547 by passing through a filter. Transistor will amplify the signal and forward it to the band-pass filter. Now the filtered signal will be amplified by a higher rate and this amplified signal will be converted to digital signal using analog to digital converter(ADC). There are many ADC available in market like CJMCU-ADS1115 which can be used. This binary data will be used by LED Driver ULN2803 to drive led according to the requirement. The blinking of LED can reach up to a speed which cannot be interpreted by human eye.

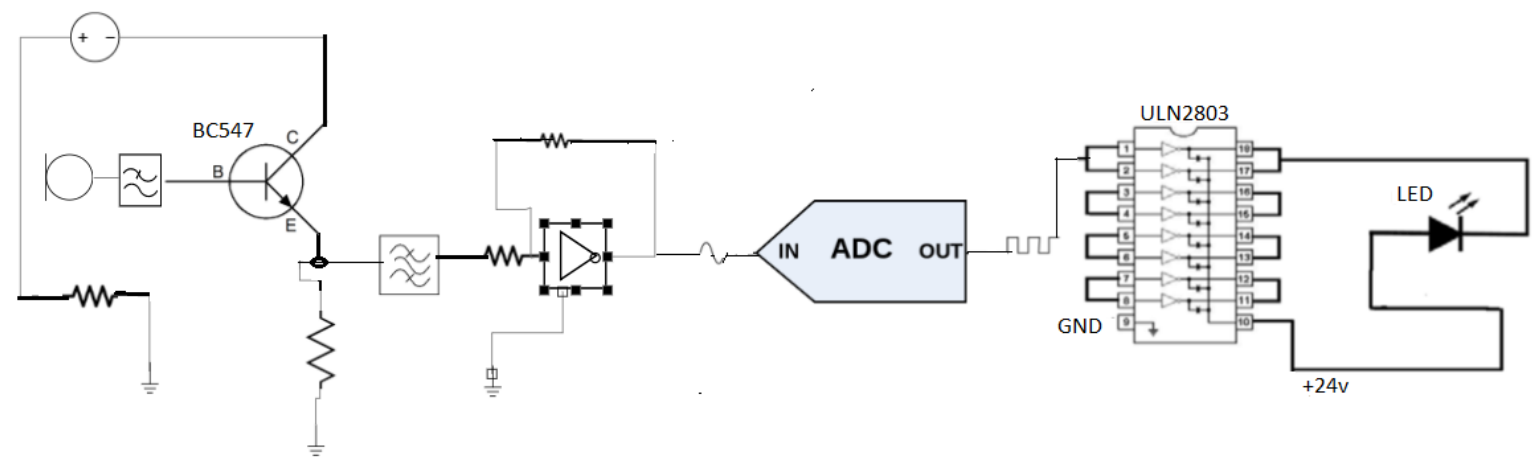

Fig:[7] Transmitter circuit for optical communication.

\subsubsection{RECEIVER SECTION}

The main characteristic of receiver section is the sensitivity of photo diode towards light. When the LED in the transmitter section will blink, photodiode will sense the light and will produce the corresponding electrical current. If the data is 1 it will generate $5 \mathrm{v}$ current for certain time and if the data is 0 it will not produce any current i.e. $0 \mathrm{~V}$. The use of photodiode will add some noise in the signal and to filter it we will use band-pass filter. The filtered signal will be very weak, so in order to make it strong we will use double stage amplification. First, we will use LM339 to amplify the signal and in second stage of amplification we will use LM386 to amplify the power up to a limit which is enough for a boned -conduction speaker to produce a sound which can be easily heard by human.

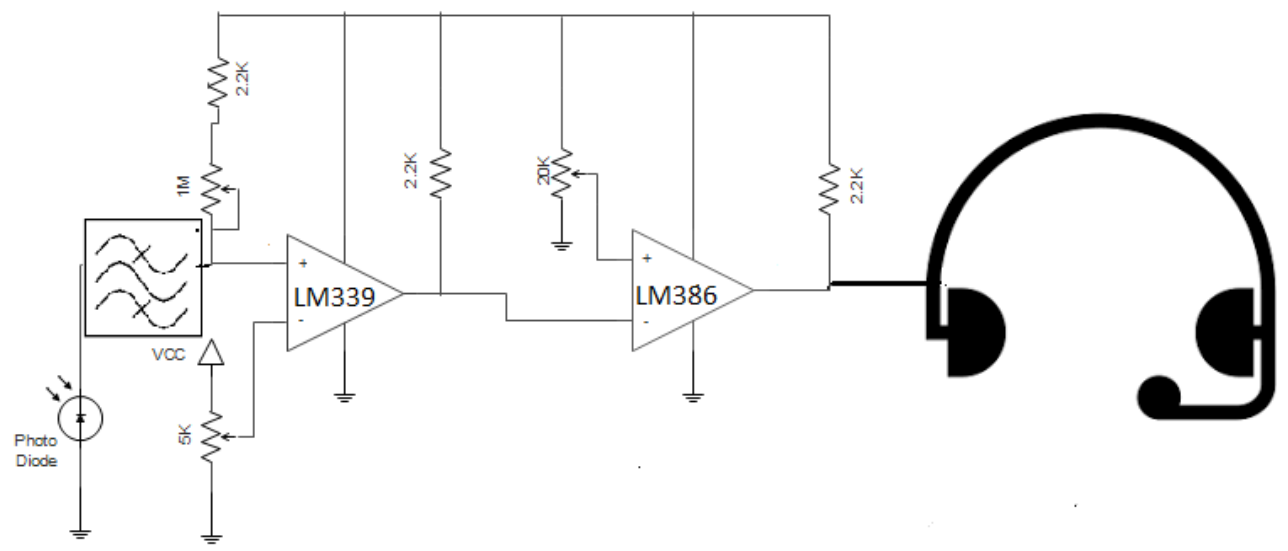

Fig:[8] Circuit of Receiver Section for optical communication. 
4. LI-FI AND WI-FI COMPARISON

\begin{tabular}{|c|c|c|}
\hline PARAMETER & LI-FI & WI-FI \\
\hline SPEED & $>1 \mathrm{~GB} / \mathrm{s}$ & AROUND $150 \mathrm{MB} / \mathrm{S}$ \\
\hline MEDIUM & VISIBLE LIGHT & RADIO SPECTRUM \\
\hline SPECTRUM RANGE & $\begin{array}{l}\text { VISIBLE LIGHT HAS } 10000 \text { TIMES } \\
\text { MORE SPECTRUM RANGE THAN } \\
\text { RADIO }\end{array}$ & $\begin{array}{l}\text { LESS SPECTRUM RANGE THAN } \\
\text { VISIBLE LIGHT }\end{array}$ \\
\hline SECURITY & $\begin{array}{l}\text { LIFI CANNOT PASS THROUGH } \\
\text { WALLS PROVIDING SECURITY }\end{array}$ & $\begin{array}{l}\text { WIFI CAN PASS THROUGHT THE } \\
\text { WALLS PROVIDING HACKERS A } \\
\text { CHANCE TO ACCESS DATA. }\end{array}$ \\
\hline COVERAGE DISTANCE & APPROXIMATELY 10 METRES & ABOUT 32 METRES \\
\hline FREQUENCY OF OPERATION & HUNDREADS OF TERA HZ & $2.4 \mathrm{GHZ}, 4.9 \mathrm{GHZ}$ AND 5GHZ \\
\hline COMPONENTS & $\begin{array}{l}\text { LAMP - DRIVERS, LED BULBS } \\
\text { AND PHOTO DETECTOR. }\end{array}$ & 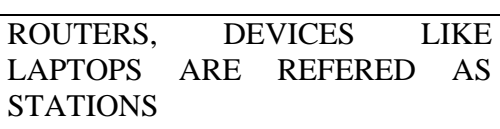 \\
\hline INTERFERNECE & $\begin{array}{llll}\text { DO NOT } & \text { HAVE INTERFERENCE } \\
\text { ISSUES } & \text { SIMILAR } & \text { TO } & \text { RADIO } \\
\text { WAVES } & & & \end{array}$ & $\begin{array}{l}\text { WILL HAVE INTERFENCE ISSUES } \\
\text { SIMILAR TO NEARBY ACCESS } \\
\text { POINTS }\end{array}$ \\
\hline MERITS/DEMERITS & $\begin{array}{l}\text { INTERFERENCE IS LESS, CAN BE } \\
\text { USED IN WATER, SPACE AS A } \\
\text { MEDIUM TO TRANSFER DATA }\end{array}$ & 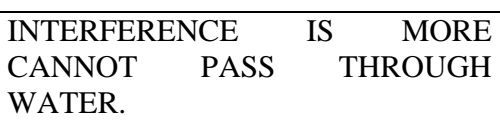 \\
\hline
\end{tabular}

\section{CONCLUSION}

Li-Fi technology is already attracting the world due its numerous advantages. Li-Fi can definitely work as a standalone communication system which can be used anywhere with high security, efficiency and speed. This technology is cost effective technology so it can be implemented even with low budgets. Li-fi will provide freedom from the harmful radio waves. It can provide alternative for almost every radio - based wireless system with easy implementation. Accessing internet, transfer of data, controlling wireless systems everything can be accomplished.

\section{FUTURE WORK}

PureLIFI has stated that each light has a unique IP address which means advanced geofencing can be deployed in a Li-Fi network that is navigation system for space can be developed which can be a revolutionary step. Self-Driving cars can communicate with each other with the help of light. Li-Fi will also be helpful in medical sciences. Indoor Positioning will also be possible with help of Li-Fi.

\section{REFERENCES}

[1] Anurag Sarkar, Prof. Shalabh Agarwal, Dr. Asoke Nath "LiFi Technology: Data Transmission through visible light" "International Journal of Advance Research in Computer Science and Management Studies" "volume 3,Issue 6, June 2015".

[2] NIKSHEP K N,SOWMYA G "voice and data communication using li-fi" International Journal of Advanced Computational Engineering and Networking, ISSN: 2320-2106, "volume-4, Issue-10, Oct.-2016" 